\title{
Symmetry studies in the nonlinear optical properties of molecular systems
}

\section{Estudios de Simetrías en las propiedades ópticas no lineales de sistemas moleculares}

\author{
J. L .Paz ${ }^{1,2 *}$, Luis G. Rodríguez ${ }^{3}$, J. R. León-Torres ${ }^{1}$, Juan F. Cárdenas ${ }^{1}$, \\ Luis Lascano ${ }^{1}$, César Costa-Vera ${ }^{1,4}$ \\ 1. Departamento de Física, Escuela Politécnica Nacional, Ladrón de Guevara, \\ E11-253, 170517, Apdo.17-12-866, Quito, Ecuador; \\ 2. Departamento de Química, Universidad Simón Bolívar, \\ Apdo 89000, Caracas 1086, Venezuela; \\ 3. Escuela de Física y Matemática, Escuela Superior Politécnica de Chimborazo, \\ Panamericana sur km 1-1/2, Riobamba-Ecuador, ec060155; \\ 4. Grupo Ecuatoriano para el Estudio Experimental y Teórico de Nanosistemas \\ Diego de Robles y Vía Interoceánica, USFQ, N104-E, Quito, Ecuador. \\ ${ }^{(*)}$ E-mail: jlpaz@usb.ve \\ Received: 04/06/2016 Accepted: 09/11/2016 \\ DOI: $10.7149 /$ OPA.49.4.49008
}

\begin{abstract}
:
Symmetries of the nonlinear optical properties (rotation, reflection, inversion) of a two-level molecular system coupled to the thermal reservoir and interacting with classical electromagnetic fields, are studied. The thermal reservoir induces a time-dependent shift of the two-level resonance frequency. A perturbative methodology based in cumulant expansions to obtain the nonlinear macroscopic polarization, is employed. These symmetry properties show the dependence of the measured of the nonlinear optical properties with the variations in the frequencies of the incident fields.
\end{abstract}

Key words: nonlinear optical properties, four-wave mixing, stochastic effects

\section{RESUMEN:}

En este trabajo hemos estudiado las propiedades de simetrías (inversión, reflexión, rotación) en las respuestas ópticas no lineales de un sistema molecular de dos niveles acoplado a un reservorio térmico e interactuando con campos electromagnéticos clásicos. El reservorio térmico induce un corrimiento dependiente del tiempo de la frecuencia natural de resonancia del sistema molecular. Una metodología perturbativa basada en expansiones de cumulantes es empleada para obtener la polarización macroscópica no lineal. Estas propiedades de simetría muestran la dependencia de la medida de las propiedades ópticas no lineales con las variaciones de frecuencia de los campos incidentes.

Palabras clave: propiedades ópticas, mezcla de cuatro ondas, efectos estocásticos.

\section{REFERENCES AND LINKS / REFERENCIAS Y ENLACES}

[1] A. Mendoza-García, A. Romero-DePablos, M.A. Ortega, J.L.Paz, L. Echevarria, "Theoretical model for the calculation of nonlinear optical properties of Gold Nanoparticles", J. Nonlinear Opt. Phys. Mater. 19, 427-443 (2010). https://doi.org/10.1142/S0218863510005297

[2] Mendoza, J.L.Paz, M. Gorayeb, A.J. Hernández, E. Castro, P. Martin, "Solvent effect in the determinations of the nonlinear optical properties", J. Nonlinear Opt. Phys. Mater. 17, 511- 520 (2008).

https://doi.org/10.1142/S0218863508004305 
[3] A. Cotton, La Teoría de grupos aplicada a la Química, México, Limusa (1977)

[4] I.A.-D.H.A. Al-Saidi, S.A.-D. Abdulkareem, "Nonlinear optical properties and optical power limiting effect of Giemsa dye", Optics \& Laser Technology 82, 150-156 (2016). https://doi.org/10.1016/i.optlastec.2016.03.013

[5] P. Sudheesh, N.K. Siji Narendran, K. Chandrasekharan, "Third-order nonlinear optical responses in derivatives of phenylhydrazone by Z-scan and optical limiting studies-influence of noble metal nanoparticles", Optical Materials 36, 304-309 (2013).

https://doi.org/10.1016/i.optmat.2013.09.014

[6] J. Niziol, J. Pielichowski, "Usability of epoxy resins in conjunction with carbazole dyes in non-linear optics applications", Optical Materials 32, 673-676 (2010). https://doi.org/10.1016/i.optmat.2010.01.035

[7] O.Y. Khyzhun, V.L. Bekenev, O.V. Parasyuk, S.P. Danylchuk, N.M. Denusyuk, A.O. Fedorchuk, N.Alzayed, I.V. Kityk, "Single crystal growth and the electronic structure of orthorhombic $\mathrm{Tl}_{3} \mathrm{PbBr}_{5}$ : A novel material for non-linear optics", Optical Materials 35, 1081-1089 (2013). https://doi.org/10.1016/i.optmat.2012.12.008

[8] K. Thirupugalmani, S. Karthick, G. Shanmugam, V. Kannan, B. Sridhar, S. Brahadeeswaran, "Secondand third-order nonlinear optical and quantum chemical studies on 2-amino-4-picolinium nitrophenolate-nitrophenol: A phasematchable organic single cristal", Optical Materials 49, 158-170 (2015). https://doi.org/10.1016/i.optmat.2015.09.014

[9] X. Li, LiLi Zou, Chenglin Liu, Zhi-Hai Zhang, Jian-Hui Yuan, "The nonlinear optical rectification and second harmonic generation in asymmetrical Gaussian potential quantum well: Effects of hydrostatic pressure, temperature and magnetic field", Optical Materials 53, 218-223 (2016). https://doi.org/10.1016/i.optmat.2016.01.043

[10] S.A. Mikhailov, "Electromagnetic nonlinearities in graphene", Europhys Lett. 79, 27002(2013). https://doi.org/10.1209/0295-5075/79/27002

[11] J.L.Cheng, N. Vermeulen and J.E. Sipe, "Third order optical nonlinearity of graphene". New Journal of Physics 16, 053014(2014). https://doi.org/10.1088/1367-2630/16/5/053014

[12] C.-B. Yao, Y.D. Zhang, J. Li, D.T. Chen, H.T. Yin, C.Q. Yu, P. Yuan, "Study of the nonlinear optical properties and behavior in phenoxy-phthalocyanines liquid at nanosecond laser pulses, Optical Materials 37, 80-86 (2014) https://doi.org/10.1016/i.optmat.2014.05.003

[13] P.J. Colmenares, J.L.Paz, R. Almeida, "On the optical stochastic Bloch equations", Physics Lett. A 199, 163-168 (1995) https://doi.org/10.1016/0375-9601(95)00081-D

[14] P.J. Colmenares, R. Almeida, J.L.Paz, "Stochastic effects of the solvent on the absorptive and dispersive processes in a two-level system up to first order in the external field", J. of Phys: At, Mol. Opt. Phys. 28, 4377-4388 (1995) https://doi.org/10.1088/0953-4075/28/19/021

[15] M. Gorayeb, J.L.Paz, A.J. Hernández. "Effect of inhomogeneous line broadening due to molecular collisions on a two-level system interacting with a high-intensity electromagnetic field", Opt. Commun. 251, 216-228 (2005) https://doi.org/10.1016/i.optcom.2005.03.030

[16] M. Gorayeb, J.L.Paz, "Effects of the spectral inhomogeneity on the optical properties in the Four-Wave mixing signal", J. Mol. Spect. 232, 157-166 (2005). https://doi.org/10.1016/i.jms.2005.04.002

[17] J.L.Paz, A. Mastrodomenico, César Costa-Vera, J. F. Cárdenas, L. G. Rodriguez, "Rotating-Wave Approximation effects on the nonlinear optical responses of complex molecular systems using a Four-Wave Mixing Signal", J. of Modern Optics 62, 403-411 (2015). https://doi.org/10.1080/09500340.2014.986235

[18] J.L.Paz, M. Izquierdo, L.G. Rodriguez, César Costa-Vera, "Propagation of the Four-Wave mixing signal in a molecular system: Stochastic considerations of the thermal bath", Opt. Pur. Appl. 48, 225-242 (2015)

https://doi.org/10.7149/OPA.48.3.225 
[19] C.W. Gardiner, Handbook of Stochastic Methods for Physics, Chemistry and Natural Sciences. Berlin, Springer (1985).

https://doi.org/10.1007/978-3-662-02452-2

[20] J.L.Paz, A. Mendoza-García, "Solvent influence on the nonlinear optical properties of molecular Systems in the presence of degenerate and non-degenerate Four-Wave Mixing", J. Mod. Opt. 59, 71$82(2012)$ https://doi.org/10.1080/09500340.2011.639962

[21] J.L.Paz, L.G. Rodríguez, César Costa-Vera, "Four-wave mixing signal intensity: relaxation time effects on the absorptive and dispersive optical properties in a two-level system", J. Nonlinear Opt. Phys. Mater. 24, 1550023 (19 pages) (2015).

https://doi.org/10.1142/S021886351550023X

[22] H.J. Franco, J.L.Paz, I. Reif, A. Marcano O, M.C. Salazar, "Symmetry properties of the Rayleigh-type optical mixing signal in frequency space", J. Opt. Soc. Am. B 7, 57-63 (1990). https://doi.org/10.1364/JOSAB.7.000057

\section{Introducción}

En los últimos años múltiples estudios se han realizado para caracterizar sistemas moleculares empleando propiedades ópticas no lineales [1,2], a través de diversas técnicas espectroscópicas. Se han desarrollado variados modelos con este fin, empleando sistemas de dos estados donde en términos generales, la estructura interna de la molécula no ha sido considerada como algo relevante. El estudio de la interacción radiación-materia a través de desarrollos teóricos, simulaciones computacionales y experimentos, permiten describir a sistemas materiales a través de sus parámetros moleculares proporcionando información de propiedades ópticas tales como los coeficientes de absorción e índice de refracción. A partir de la invención del láser se ha dado mayor importancia a los fenómenos no lineales que tienen lugar bajo la presencia de intensos campos electromagnéticos, y es precisamente la respuesta no lineal del sistema la que permite reconocer el potencial de ciertos materiales para aplicaciones en el desarrollo de dispositivos no lineales, optoelectrónicos y fotónicos. Los colorantes orgánicos y algunos materiales cristalinos han sido extensamente estudiados, ya que éstos muestran la más alta respuesta óptica no lineal, debido a sus propiedades químicas asociadas a los tipos de enlace y a propiedades cristalinas asociadas con los diferentes grupos de simetría [3]. De forma particular se analiza la susceptibilidad a $2^{\circ}$ y $3^{\text {er }}$ orden, ya que su estudio ofrece información sobre los distintos procesos de dispersión que tienen lugar entre el material y el campo electromagnético. Al Saidi y colaboradores [4] han reportado cambios en las propiedades ópticas respecto a la concentración molecular del colorante estudiado. La respuesta que arroja el sistema al cambio de la concentración molecular se presenta a través de la alta absorción saturable no lineal medida, y esta característica se ha explotado a través del desarrollo de dispositivos no lineales como limitadores ópticos. Otro aspecto importante en el campo de las propiedades ópticas no lineales es inducir la respuesta no lineal a través de la funcionalización del material con agentes externos. Sudheesh y colaboradores [5] han logrado inducir a través de nanopartículas de Oro y Plata una alta respuesta óptica a tercer orden en la estructura de Phenylhydrazone, lo cual ha demostrado que la respuesta no lineal ha sido producida a través de la interacción entre el campo electromagnético y los electrones donados por los metales.

Los procesos multi-fotónicos que tienen lugar en la interacción radiación-materia son de vital importancia ya que permiten el estudio de los cambios en las propiedades ópticas, en particular del índice de refracción. Niziol y colaboradores [6] realizaron medidas en la susceptibilidad a $2^{\circ}$ y $3^{\text {er }}$ orden del colorante orgánico Azocarbazole, con las cuales estudiaron fenómenos ópticos como el efecto Pockels y el efecto Kerr, los mismos que están asociados al cambio en el índice de refracción. Las propiedades ópticas no lineales también han sido ampliamente estudiadas en cristales, debido a la relación que presentan sus características de simetría con las susceptibilidades de alto orden. Así por ejemplo, Khyzum y colaboradores [7] han estudiado la respuesta no lineal en $\mathrm{TI}_{3} \mathrm{PbBr}_{5}$ asociándola a la simetría y a los enlaces químicos que presenta el cristal. En la investigación realizada por Thirupugalmani y colaboradores [8] se realizaron pruebas de daño óptico en materiales cristalinos, y estudios de la susceptibilidad a $3^{\text {er }}$ orden que permitieron proponer una aplicación en limitadores ópticos.

En el aspecto teórico, varias investigaciones se llevaron a cabo bajo el formalismo de la matriz de densidad para analizar el sistema material, lo que ha permitido estudiar las susceptibilidades ópticas, la conductividad eléctrica y la polarizabilidad [9-11]; en particular, en [11, 12] se analizan las propiedades 
ópticas del grafeno que dan cuenta de los procesos multi-fotónicos que ocurren en su estructura de bandas y se ven reflejadas en los cambios de la conductividad eléctrica. Estas mismas investigaciones han permitido explotar el potencial del grafeno en el desarrollo de dispositivos optoelectrónicos, tales como moduladores ópticos, limitadores ópticos, fotodetectores y absorbentes saturables por mencionar algunos ejemplos.

En este trabajo estudiamos las propiedades ópticas de un colorante orgánico (Malaquita Verde) a través de la respuesta no lineal que produce el material al interaccionar con dos campos electromagnéticos intensos, un haz de bombeo y un haz de prueba, todo ello en el marco de la señal óptica de mezcla de cuatro ondas. Siguiendo trabajos anteriores, nuestro desarrollo considera un sistema de dos estados interactuando con un reservorio térmico aleatorio. En el formalismo de la matriz densidad se evalúan las distintas componentes perturbadas, tanto de coherencias como de poblaciones, y con ello la polarización no lineal macroscópica inducida. Luego, haciendo uso de las ecuaciones de Maxwell determinamos expresiones analíticas para las propiedades ópticas absortivas y dispersivas y estudiamos el comportamiento de las propiedades ópticas en el espacio $\varepsilon\left(\Delta_{1}, \Delta_{2}\right)$ (donde $\Delta_{j}=\omega_{j}-\omega_{0}, \mathrm{j}=1,2$ ) de las frecuencias ópticas a través de un mapa de contorno. A partir de ciertas coordenadas específicas que representan 4 puntos dispuestos simétricamente en torno al eje $\Delta_{1}=\Delta_{2}$, evaluamos los cambios que tienen las propiedades ópticas frente a propiedades de inversión, reflexión y ejes de rotación impropios. Los resultados muestran que hay modificaciones en las propiedades de simetría con cambios específicos simultáneos de las variables $\Delta_{1} \mathrm{y} \quad \Delta_{2}$. Estas posibles asimetrías en los espectros de absorción imposibilitan la determinación de los tiempos de relajación, comúnmente hallados de los semi-anchos a la semi-altura (HWHM) en tales perfiles ópticos.

\section{Teoría}

En el presente estudio hemos considerado la evolución temporal de un sistema de dos niveles $|a\rangle$ y $|b\rangle$ interactuando con un reservorio térmico estocástico y con campos electromagnéticos clásicos, de acuerdo con la expresión Liouvilliana de la forma:

$$
\dot{\rho}_{\xi}(t)=A_{\xi}(t) \rho_{\xi}(t)+R
$$

$\rho_{\xi}(t)$ es la matriz densidad asociada al conjunto de dos estados, $A_{\xi}(t)$ es la matriz que contiene todos los mecanismos radiativos propios de la interacción radiación-materia, con dependencia en la variable estocástica, mientras que $\mathrm{R}$ representa la matriz de relajación, asociada a los mecanismos disipativos que tienen lugar entre el subsistema de estudio y el ambiente térmico. En nuestro estudio, definimos $\xi$ como una variable estocástica que representa los efectos colisionales del baño térmico con el sistema activo, dada por $\xi(t)=\omega_{0}+\sigma(t)$, donde $\omega_{0}$ representa la frecuencia natural de resonancia del sistema de dos estados. En el término $\sigma(t)$ se depositan todos los aspectos relacionados con la dinámica aleatoria [1317]. Notemos que la frecuencia natural determinista ha sido desplazada a la función temporal $\xi(t)$ como una función estocástica. La ec. (1) es, en sí, la llamada ecuación óptica de Bloch estocástica (OSBE) para la matriz densidad en el conjunto de estados $|a\rangle$ y $|b\rangle$. Es de interés en lo que sigue, estudiar la señal de mezcla de cuatro ondas (FWM), la cual surge de un proceso de dispersión de un haz fuerte de bombeo con la red de oscilaciones que se forma entre el haz fuerte de bombeo y un haz de prueba de intensidad menor. Si esta señal se observa a través de un proceso de acoplamiento entre los haces involucrados, el orden mínimo de aparición es el tercero [1,2], donde el haz de bombeo es tratado a segundo orden, mientras que el haz de prueba es tratado al primer orden. Trabajos equivalentes, pero considerando efectos de saturación a más alto orden del campo intenso, han sido presentados previamente con la finalidad de estudiar la propagación a lo largo del camino óptico en señales de mezcla de cuatro ondas [18]. Haciendo tales desarrollos en teoría de perturbaciones para los elementos de la matriz densidad, tanto de las coherencias como de las poblaciones de los dos estados, la ecuación anterior queda de la forma:

$$
\dot{\tilde{\rho}}_{\xi}(t)=\tilde{A}_{\xi}(t) \tilde{\rho}_{\xi}(t)+\tilde{\mathrm{R}}
$$




$$
\tilde{\rho}_{\xi}(t)=\left(\begin{array}{c}
\rho_{b a}^{(3)}(t) \\
\rho_{D}^{(2)}(t) \\
\rho_{b a}^{(1)}(t) \\
\rho_{a b}^{(1)}(t)
\end{array}\right) ; \tilde{A}_{\xi}(t)=\left(\begin{array}{cccc}
-\xi_{t} & -\frac{i}{\hbar}\left(H_{I}\right)_{b a} & 0 & 0 \\
0 & -\frac{1}{T_{1}} & \frac{2 i}{\hbar}\left(H_{I}\right)_{a b} & -\frac{2 i}{\hbar}\left(H_{I}\right)_{b a} \\
0 & 0 & -\xi_{t} & 0 \\
0 & 0 & 0 & -\xi_{t}^{*}
\end{array}\right) ; \tilde{R}=\frac{i}{\hbar}\left(\begin{array}{c}
0 \\
0 \\
-\left(H_{I}\right)_{b a} \\
\left(H_{I}\right)_{a b}
\end{array}\right) \rho_{D}^{(0)}
$$

donde, $\xi_{t} \equiv i \xi(t)+1 / T_{2}$, con $\mathrm{T}_{1}$ y $\mathrm{T}_{2}$ definidos como los tiempos de relajación longitudinal y transversal, respectivamente; $\rho_{D}(t) \equiv \rho_{a a}(t)-\rho_{b b}(t)$ es la diferencia de las poblaciones entre los dos estados; $\rho_{D}^{(0)}$ es el valor de equilibrio de la diferencia poblacional en ausencia de la perturbación; el superíndice (n) que hemos colocado en cada uno de los elementos de la matriz $\tilde{\rho}_{\xi}(t)$ establece el orden, relacionado con la amplitud de los campos electromagnéticos considerados. La interacción radiación-materia en la representación de interacción se define a través del Hamiltoniano, dado por $H_{I}=-\vec{\mu} . \vec{E}(t)$, donde $\vec{\mu}$ es el vector de momento dipolar, mientras que $\vec{E}(t)$ representa la amplitud del campo total, dado por $\vec{E}=\vec{E}_{1}+\vec{E}_{2}$, donde los subíndices 1 y 2 están referidos a los haces de bombeo y prueba, respectivamente. Resolviendo la parte homogénea (H) de la ec. (2), obtenemos:

$$
\begin{gathered}
\tilde{\tilde{\rho}}_{\xi}(t)=\tilde{A}_{\xi}^{H}(t) \tilde{\rho}_{\xi}(t)+\tilde{R}^{H} \\
\tilde{A}_{\xi}^{H}(t)=\left(\begin{array}{cccc}
0 & -\frac{i}{\hbar}\left(H_{I}\right)_{b a} f_{1} f_{\xi} & 0 & 0 \\
0 & -\frac{1}{T_{1}} & \frac{2 i}{\hbar}\left(H_{I}\right)_{a b} f_{2} & -\frac{2 i}{\hbar}\left(H_{I}\right)_{b a} f_{2} \\
0 & 0 & 0 & 0 \\
0 & 0 & 0 & 0
\end{array}\right) ; \tilde{R}^{H}=\frac{i}{\hbar}\left(\begin{array}{c}
0 \\
0 \\
-\left(H_{I}\right)_{b a} f_{1} f_{\xi} \\
\left(H_{I}\right)_{a b} f_{1} f_{\xi}^{*}
\end{array}\right) \rho_{D}^{(0)} \\
\operatorname{con} f_{k}=\exp \left[\frac{1}{T_{k}}\left(t-t_{0}\right)\right] ; f_{\xi}=\exp \left(\int_{t_{0}}^{t} i \xi(t) d t\right) \mathrm{k}=1,2
\end{gathered}
$$

Sustituyendo la forma del Hamiltoniano de interacción en la expresión para la coherencia a primer orden, tenemos:

$$
\rho_{b a}^{(1)}(t)=i \Omega_{1} \rho_{D}^{(0)} \exp \left(-\frac{t}{T_{2}}\right) \exp \left(i k_{1} r\right) \int_{-\infty}^{t} \exp \left(\int_{t}^{y} i \xi(s) d s\right) \exp \left(\frac{y}{T_{2}}\right) \exp \left(-i \omega_{1} y\right) d y
$$

donde $\Omega_{j}=\vec{\mu} \cdot \vec{E}_{j} / \hbar$ es la frecuencia de Rabí y $\vec{E}_{j}(t)=\vec{E}_{j 0} \exp \left(i \vec{k}_{j} \cdot \vec{r}-\omega_{j} t\right)$ es la componente j del campo. En esta expresión para la coherencia a primer orden, hemos supuesto que se cumple la condición $\rho_{b a}^{(1)}(-\infty)=0$ Usando la ec. (3), obtenemos la expresión para la componente $\rho_{D}^{(2)}(t)$, dada por:

$$
\begin{gathered}
\rho_{D}^{(2)}(t)=2 \Omega_{1} \Omega_{2}^{*} \rho_{D}^{(0)} \exp \left(-\frac{t}{T_{1}}\right) \exp \left[i\left(\mathrm{k}_{1}-k_{2}\right) r\right]\left\{\Psi_{1}(t)+\Psi_{2}(t)\right\} \\
\Psi_{1}(t)=\int_{-\infty}^{t} d z \exp \left[\left(T_{1}^{-1}-T_{2}^{-1}+i \omega_{2}\right) z\right] \int_{-\infty}^{t} \exp \left(\int_{z}^{y} i \xi(s) d s\right) \exp \left(\frac{y}{T_{2}}\right) \exp \left(-i \omega_{1} y\right) d y \\
\Psi_{2}(t)=\int_{-\infty}^{t} d z \exp \left[\left(T_{1}^{-1}-T_{2}^{-1}-i \omega_{1}\right) z\right] \int_{-\infty}^{t} \exp \left(-\int_{z}^{y} i \xi(s) d s\right) \exp \left(\frac{y}{T_{2}}\right) \exp \left(i \omega_{2} y\right) d y
\end{gathered}
$$


Finalmente, la coherencia a tercer orden responsable de la generación de la señal de mezcla de cuatro ondas (FWM), se obtiene sustituyendo la expresión anterior para $\rho_{D}^{(2)}(t)$ en la expresión para $\rho_{b a}^{(3)}(t)$ proveniente de la matriz, ec. (3):

$$
\begin{gathered}
\rho_{b a}^{(3)}(t)=\mathrm{K}(\mathrm{r}, \mathrm{t}) \mathrm{I}_{1}(t)\left\{\Phi_{1}+\Phi_{2}\right\} \\
K(r, t)=2 i \Omega_{1}^{2} \Omega_{2}^{*} \rho_{D}^{(0)} \exp \left(-\frac{t}{T_{2}}\right) \exp \left[i\left(2 k_{1}-k_{2}\right) r\right] \\
\Phi_{1}=I_{2}(x) I_{1}(z) \exp \left(\int_{t}^{x} i \xi(q) d q\right) \exp \left(\int_{z}^{y} i \xi(s) d s\right) \\
\Phi_{2}=I_{1}^{*}(x) I_{2}^{*}(z) \exp \left(\int_{t}^{x} i \xi(q) d q\right) \exp \left(-\int_{z}^{y} i \xi(s) d s\right) \\
I_{1}(t)=\int_{-\infty}^{t} d x \exp \left(-x B_{1}\right) ; I_{n}(x)=\int_{-\infty}^{x} d z \exp \left(\mathrm{z} B_{n}\right) ; I_{m}(\mathrm{z})=\int_{-\infty}^{z} d y \exp \left(\mathrm{yA}_{m}\right),
\end{gathered}
$$

Aquí, $A_{k}=\left(T_{2}^{-1}\right)-i \omega_{k}$ y $B_{k}=\left(T_{1}^{-1}\right)-A_{k}$. En la expresión para la coherencia se requiere tomar el valor promedio sobre todas las realizaciones de la variable aleatoria en el conjunto estadístico:

$$
\left\langle\rho_{b a}^{(3)}(t)\right\rangle_{\xi}=K(r, t) I_{1}(x, t)\left\{\left\langle\Phi_{1}\right\rangle_{\xi}+\left\langle\Phi_{2}\right\rangle_{\xi}\right\}
$$

donde

$$
\begin{gathered}
\left\langle\Phi_{1}\right\rangle_{\xi}=I_{2}(z, x) I_{1}(y, z)\left\langle\exp \left(\int_{t}^{x} i \xi(q) d q\right) \exp \left(\int_{z}^{y} i \xi(s) d s\right)\right\rangle_{\xi} \\
\left\langle\Phi_{2}\right\rangle_{\xi}=I_{1}^{*}(z, x) I_{2}^{*}(y, z)\left\langle\exp \left(\int_{t}^{x} i \xi(q) d q\right) \exp \left(-\int_{z}^{y} i \xi(s) d s\right)\right\rangle_{\xi}
\end{gathered}
$$

Es ahora necesario resolver el valor promedio en cada una de las funciones $\left\langle\Phi_{k}\right\rangle_{\xi}$. De acuerdo con la forma que cada una de ellas presenta, en términos de esas integrales estocásticas, podemos emplear un desarrollo de cumulantes, donde el valor promedio de la función que depende de las variables estocásticas, es determinado como una función del promedio de la variable a distintos órdenes [19], esto es:

$$
\langle\exp (\operatorname{imx}(t))\rangle=\exp \left\{\operatorname{im}\left\langle\left\langle x\left(t_{1}\right)\right\rangle\right\rangle+\frac{i^{2} m^{2}}{2 !}\left\langle\left\langle x\left(t_{1}\right) x\left(t_{2}\right)\right\rangle\right\rangle+\ldots+\frac{i^{n} m^{n}}{n !}\left\langle\left\langle x\left(t_{1}\right) \ldots x\left(t_{n}\right)\right\rangle\right\rangle\right\}
$$

Para un proceso Gaussiano, sólo se hacen distintos de cero el primer y segundo cumulante, siendo el de primer orden el asociado al valor promedio de la variable aleatoria, que por el teorema de límite central queda definido como $\langle\xi\rangle=\omega_{0}$, mientras que el segundo cumulante está asociado a la función de correlación $\left\langle\xi\left(t_{1}\right) \xi\left(t_{2}\right)\right\rangle$. Usando para esta correlación un proceso dado por una función de ruido blanco $\left\langle\xi\left(t_{1}\right) \xi\left(t_{2}\right)\right\rangle=2 \gamma \delta\left(t_{1}-t_{2}\right)$, el valor promedio de la coherencia temporal a tercer orden, está dado por:

$$
\left\langle\rho_{b a}^{(3)}(t)\right\rangle_{\xi}=\left\langle\rho_{b a}^{(3)}\left(\omega_{3}\right)\right\rangle_{\xi} \exp \left(-i \omega_{3} t\right)
$$

mientras que la componente de Fourier $\left\langle\rho_{b a}^{(3)}\left(\omega_{3}\right)\right\rangle_{\xi}$ en la aproximación de la onda rotante [17] viene dada por: 


$$
\left\langle\rho_{b a}^{(3)}\left(\omega_{3}\right)\right\rangle_{\xi}=2 i \Omega_{1}^{2} \Omega_{2}^{*} \rho_{D}^{(0)}\left\{\frac{1}{\tilde{D}_{1}} \frac{1}{\tilde{D}_{3}}+\frac{1}{D_{2}^{*}} \frac{1}{D_{3}}\right\} \frac{1}{\Gamma_{1}(\Delta)} \exp \left(i k_{3} r\right)
$$

Se introducen las resonancias $\tilde{D}_{k}=\left(\tilde{T}_{2}\right)^{-1}+i\left(\omega_{0}-\omega_{k}\right) \mathrm{y} \quad D_{k}=\left(T_{2}\right)^{-1}+i\left(\omega_{0}-\omega_{k}\right)$, con $\mathrm{k}=1,2,3$; $\Gamma_{1}(\Delta)=T_{1}^{-1}-i \Delta ; \Delta=\omega_{1}-\omega_{2}, \tilde{T}_{2}^{-1}=T_{2}^{-1}+2 \gamma$ es el tiempo de relajación efectivo, dependiente de la intensidad del ruido $\gamma$. Es necesario destacar el hecho que para la evaluación de esta componente de frecuencia, hemos considerado un desarrollo perturbativo, considerando el haz de bombeo de alta intensidad al segundo orden, mientras que el haz de prueba de menor intensidad, al primer orden. La polarización inducida macroscópica no lineal está dada por:

$$
P\left(\omega_{3}\right)=N\left\langle\left\langle\rho_{b a}^{(3)}\left(\omega_{3}\right)\right\rangle_{\xi}\right\rangle_{\theta}
$$

En esta expresión, el corchete angular externo denota el promedio sobre todas las posibilidades de orientaciones espaciales de la molécula, mientras que el corchete angular interno denota el valor promedio de todas las realizaciones de la variable aleatoria en el conjunto estadístico; $\mathrm{N}$ es la concentración total de moléculas activas en la solución química, expresada en milimolar (mM). Considerando las aproximaciones escalar y de estado estacionario, y tomando en cuenta las propiedades tensoriales en la respuesta óptica, tenemos:

$$
P\left(\omega_{3}\right)=\chi^{S V}\left(\omega_{3}\right) E\left(\omega_{3}\right)+\chi^{(3)}\left(\omega_{3}\right) E^{2}\left(\omega_{1}\right) E^{*}\left(-\omega_{2}\right)
$$

$\chi^{S V}\left(\omega_{3}\right)$ es la susceptibilidad del solvente a la frecuencia $\omega_{3}$, mientras que $\chi^{(3)}\left(\omega_{3}\right)$ es la componente de la susceptibilidad asociada a los procesos de acoplamiento que tienen lugar entre los campos incidentes. Usando ecuaciones de Maxwell para describir la variación de las amplitudes de los campos electromagnéticos conforme ocurre la propagación óptica a lo largo del eje z tenemos, en la aproximación de la envolvente suave, la siguiente expresión para el haz de la señal de mezcla de cuatro ondas:

$$
\frac{d E\left(\omega_{3}\right)}{d z}=-\alpha^{(3)}\left(\omega_{3}\right) E\left(\omega_{3}\right)
$$

$\alpha^{(3)}\left(\omega_{3}\right)$ es el coeficiente de absorción no lineal del haz de la señal de mezcla de cuatro ondas (FWM) evaluado a la frecuencia $\omega_{3}$, dado por:

$$
\alpha^{(3)}\left(\omega_{3}\right)=\frac{2 \pi \omega_{3}}{\eta^{(3)}\left(\omega_{3}\right) c} \operatorname{Im} \chi^{(3)}\left(\omega_{3}\right)
$$

$\eta^{(3)}\left(\omega_{3}\right)=\left[\eta_{0}^{2}+4 \pi \operatorname{Re} \chi^{(3)}\left(\omega_{3}\right)\right]^{1 / 2}$, siendo $\eta_{0}$ el índice de refracción del solvente (SV). Con los reemplazos correspondientes, finalmente tenemos para el coeficiente de absorción e índice de refracción, ambos a la frecuencia $\omega_{3}$, las siguientes expresiones,

$$
\begin{aligned}
& \alpha^{(3)}\left(\omega_{3}\right)=\frac{W_{1}}{\eta^{(3)}\left(\omega_{3}\right)}\left[\frac{1+T_{1} T_{2}\left(\Delta_{2} \Delta_{3}-\Delta_{3} \Delta+\Delta_{2} \Delta\right)}{a_{1} L_{2} L_{\Delta} L_{3}}+\frac{1-T_{1} \tilde{T}_{2}\left(\Delta_{1} \Delta+\Delta_{3} \Delta\right)-\tilde{T}_{2} \tilde{T}_{2} \Delta_{1} \Delta_{3}}{a_{2} \tilde{L}_{1} L_{\Delta} \tilde{L}_{3}}\right] \\
& \eta^{(3)}\left(\omega_{3}\right)=\left\{\eta_{0}^{2}+W_{2}\left[\frac{T_{2}\left(\Delta_{2}-\Delta_{3}\right)-T_{1} \Delta-T_{1} T_{2} T_{2} \Delta \Delta_{2} \Delta_{3}}{a_{1} L_{2} L_{\Delta} L_{3}}+\frac{T_{1} \tilde{T}_{2} \tilde{T}_{2} \Delta_{1} \Delta \Delta_{3}-\tilde{T}_{2}\left(\Delta_{1}+\Delta_{3}\right)-T_{1} \Delta}{a_{2} \tilde{L}_{1} L_{\Delta} \tilde{L}_{3}}\right]\right\}^{1 / 2}
\end{aligned}
$$

En las que $W_{1}=\frac{4 \pi \omega_{3} N\left|\mu_{b a}\right|^{4} \rho_{D}^{(0)}}{\hbar^{3} c}, W_{2}=\frac{8 \pi N\left|\mu_{b a}\right|^{4} \rho_{D}^{(0)}}{\hbar^{3}}$ y $\Delta_{k}=\omega_{k}-\omega_{0}$, donde éste último factor se refiere al factor de desintonización entre el campo de frecuencia $\omega_{k}(\mathrm{k}=1,2,3)$ y la frecuencia natural de resonancia del sistema de dos estados $\omega_{0}$. Hemos definido por otra parte: 


$$
\tilde{L}_{k}=\left(\tilde{T}_{2}^{-1}\right)^{2}+\left(\Delta_{k}\right)^{2} ; L_{k}=\left(T_{2}^{-1}\right)^{2}+\left(\Delta_{k}\right)^{2} \text { con } \mathrm{k}=1,2,3 \text { y } L_{\Delta}=\left(T_{1}^{-1}\right)^{2}+\Delta^{2}
$$

Es importante resaltar el hecho que las expresiones (15) y (16) constituyen el resultado central de nuestro estudio, las cuales serán el punto de partida para el análisis de las propiedades de simetrías tanto del coeficiente de absorción no lineal como del índice de refracción no lineal en sistemas moleculares complejos.

\section{Propiedades ópticas no lineales}

Para el estudio de las propiedades de simetría de los coeficientes de absorción e índices de refracción en el denominado espacio de frecuencias $\varepsilon\left(\alpha, \Delta_{1}, \Delta_{2}\right)$ y $\tilde{\varepsilon}\left(\eta, \Delta_{1}, \Delta_{2}\right)$, respectivamente, empleamos como sistema de estudio el colorante orgánico Malaquita Verde, el cual es altamente resonante y exhibe respuestas no lineales cuando interactúa con campos electromagnéticos de elevada intensidad, como es nuestro caso $[20,21]$. Las características de este colorante son, entre otras:

$$
T_{1} \approx T_{2}=1.1 \times 10^{-12} \mathrm{~s}, \mu_{a b} \approx 2.81 \times 10^{-18} \mathrm{erg}^{1 / 2} \mathrm{~cm}^{3 / 2}, \omega_{0}=3.06 \times 10^{15} \mathrm{~s}^{-1} .
$$

Las Figs. 1 y 2 muestran superficies del coeficiente de absorción no lineal y del índice de refracción no lineal, respectivamente, como función de las desintonizaciones de los campos de bombeo y de prueba, $\Delta_{1}$

y $\Delta_{2} \quad\left(s^{-1}\right)$, conforme las expresiones (15) y (16), sujetas a los parámetros moleculares del colorante orgánico empleado como sistema de estudio. El máximo de absorción se extiende alrededor del eje $\Delta_{1}=\Delta_{2}$, mientras que para el eje $\Delta_{1}=-\Delta_{2}$ hay un decaimiento de intensidad. Puede notarse igualmente en este perfil que hay zonas fuera de la región de resonancia donde parecieran darse fenómenos de amplificación en lugar de absorción $\left(\Delta_{1}= \pm 0.25 \times 10^{11} s^{-1}\right)$. Para la propiedad óptica dispersiva (Fig. 2), se nota claramente la asimetría alrededor del valor $\eta_{0}=1.333$ correspondiente al solvente empleado (agua). Puede notarse en este caso, que aunque hay un cambio de perfil óptico, los valores máximos y mínimos de esta propiedad fluctúan con una diferencia de $2 \times 10^{-3}$.

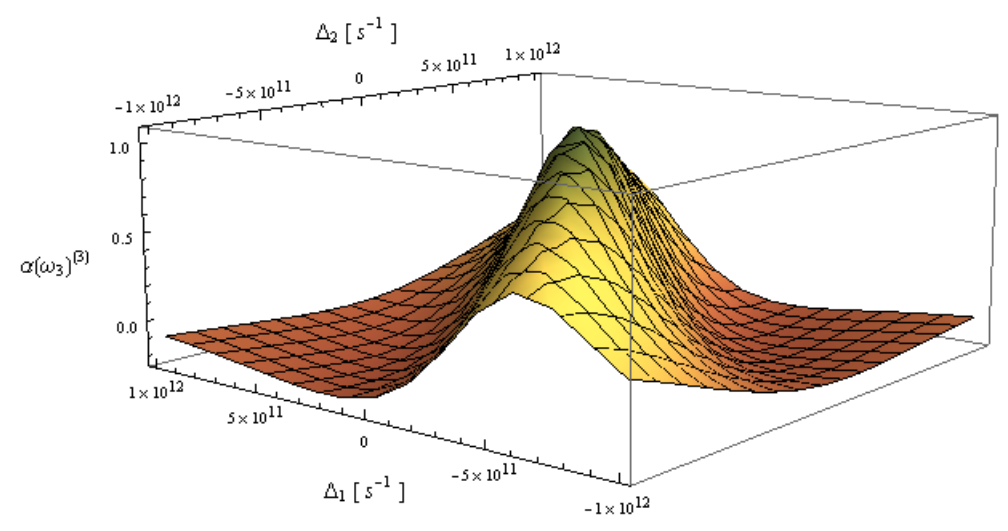

Fig. 1. Superficie del Coeficiente de absorción no lineal $\alpha\left(\omega_{3}\right)^{(3)}$ 


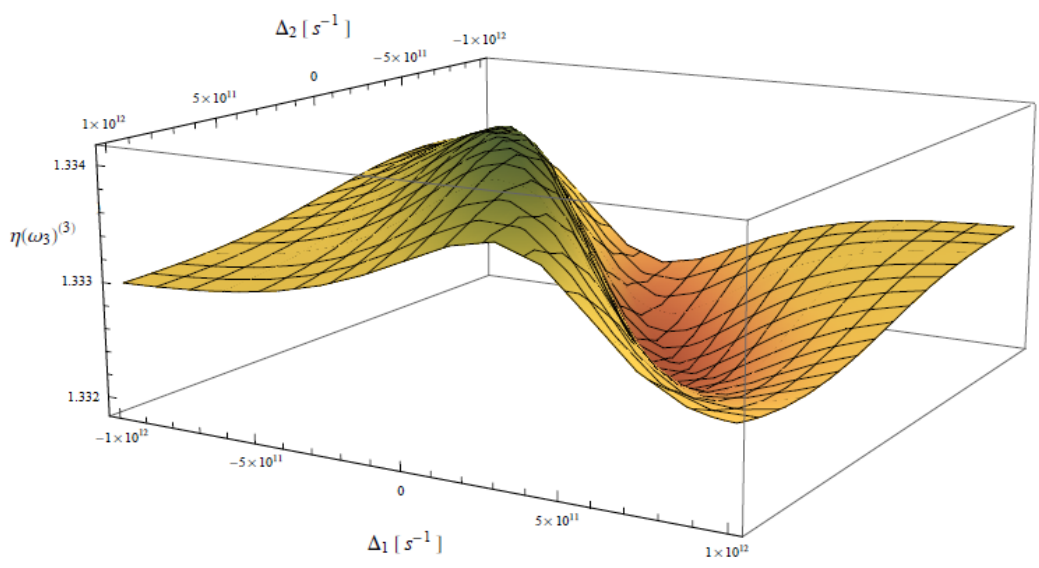

Fig. 2. Superficie del índice de refracción no lineal $\eta\left(\omega_{3}\right)^{(3)}$

Para el estudio de las propiedades de simetrías de ambas propiedades ópticas no lineales, son más convenientes las representaciones en curvas de contorno en los espacios $\varepsilon\left(\Delta_{1}, \Delta_{2}\right)$. Las Figs. 3 y 4 , provenientes de las expresiones (15) y (16), representan las curvas de nivel de ambas propiedades ópticas en función de los cambios de frecuencia tanto de bombeo $\left(\Delta_{1}\right)$ como de prueba $\left(\Delta_{2}\right)$.

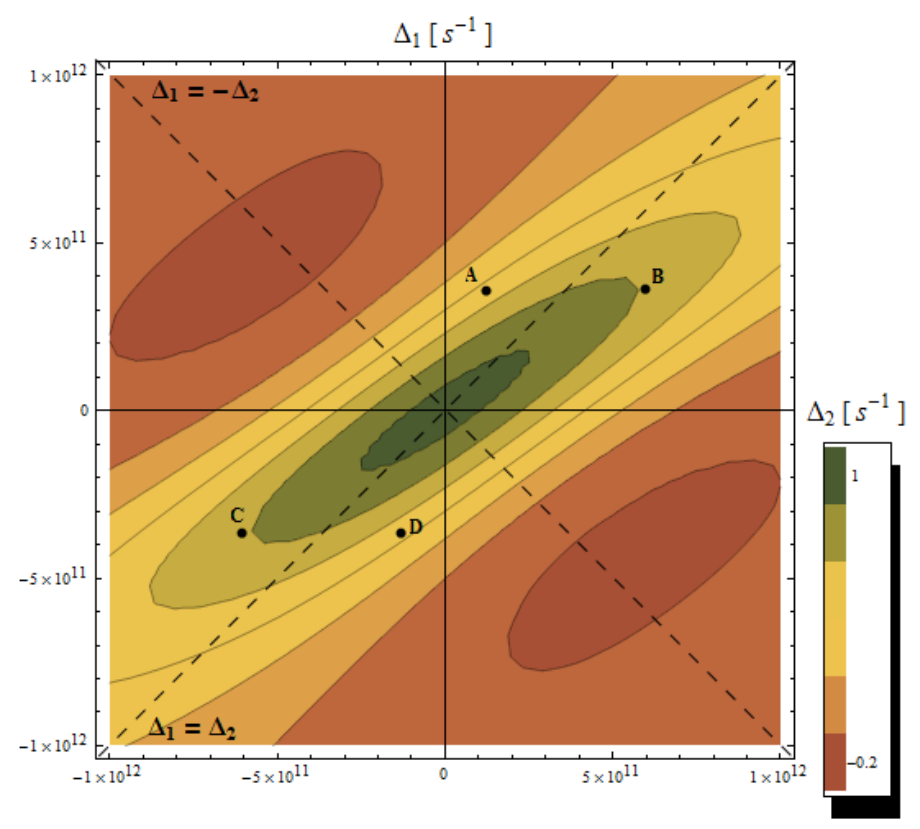

Fig. 3 Curva de contorno del coeficiente de absorción no lineal. 


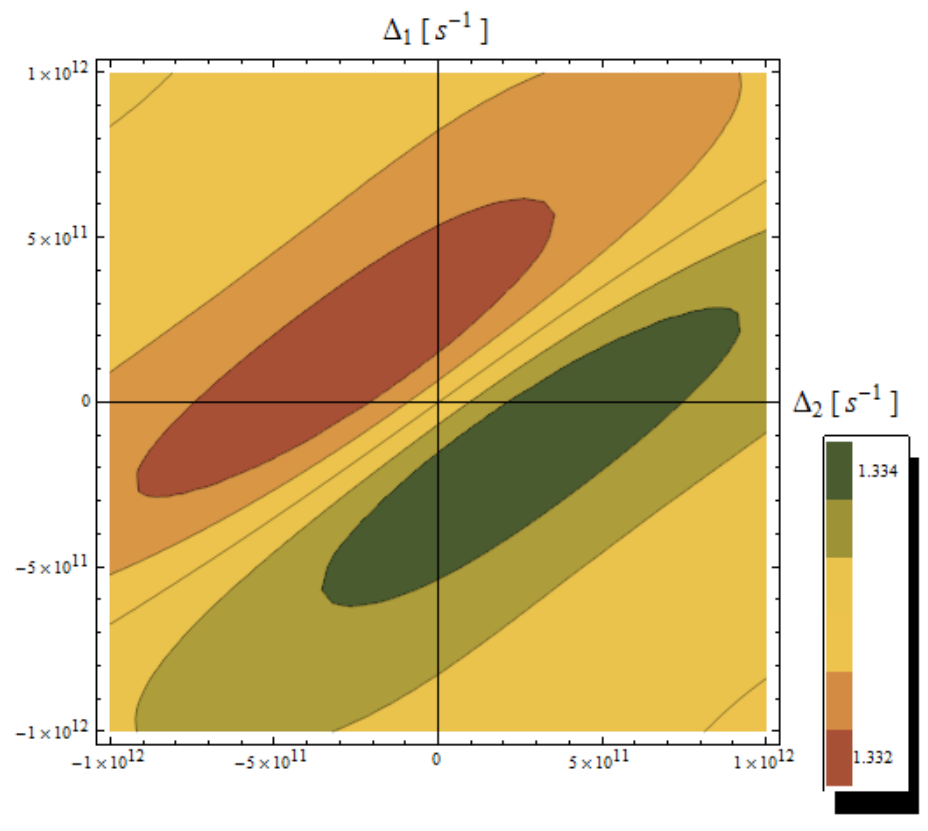

Fig. 4 Curva de contorno del Índice de refracción no lineal.

Dado que nos interesa particularmente las propiedades de simetría del perfil absortivo, hemos seleccionado los siguientes tipos de espectros: diagonales los de tipo $\Delta_{1}=\Delta_{2}$ y los no diagonales los de tipo $\Delta_{1}=-\Delta_{2}$. Hemos representado en este diagrama de contorno (Fig. 3), los puntos A, B, C, y D con características muy particulares en términos de intercambios de frecuencias claramente definidos. Las propiedades de simetría serán aquellas que podamos determinar con respecto a inversión, reflexiones o rotaciones alrededor del eje diagonal.

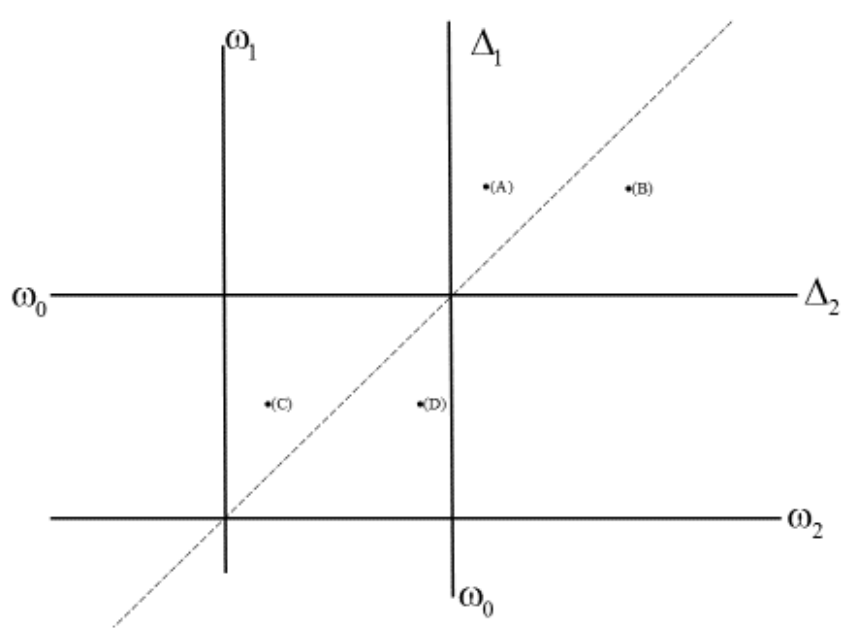

Fig. 5. Sistema de coordenadas $\left(\Delta_{1}, \Delta_{2}\right)$ para describir las propiedades de simetría en las propiedades ópticas.

Para el estudio de las propiedades de simetría en el espacio $\varepsilon$, objeto del presente trabajo, es conveniente expresar las propiedades ópticas no lineales dadas por las ecs. (15 y 16), en términos de las variables $\Delta_{1}$ y $\Delta_{2}$, de acuerdo a la Fig. 5 [22]. La Fig. 5 se corresponde con la Fig. 3, donde hemos señalado los puntos de estudio, pero además el sistema de referencia $\left(\omega_{1}, \omega_{0}\right)$ y $\left(\omega_{2}, \omega_{0}\right)$. La ec. (15) se expresa en función de las variables asociadas a $\varepsilon$ de acuerdo a la siguiente expresión: 


$$
\alpha^{(3)}\left(\Delta_{1}, \Delta_{2}\right)=\frac{W_{1}}{\eta^{(3)}}\left[H\left(\Delta_{1}, \Delta_{2}\right)\right] \text { con } H\left(\Delta_{1}, \Delta_{2}\right)=h_{0}+h_{1} \Delta_{1} \Delta+h_{2} \Delta_{1}^{2}+h_{3} \Delta^{2}
$$

los coeficientes $h_{k}\left(\Delta_{1}, \Delta_{2}\right)(\mathrm{k}=0, \ldots, 3)$ están dados por:

$$
\begin{gathered}
h_{0}=\frac{1}{a_{1}} \vartheta_{23}+\frac{1}{a_{2}} \tilde{\vartheta}_{13} ; h_{1}=\frac{6}{T_{2}} \vartheta_{23}+\frac{4 T_{2}}{\tilde{T}_{2} \tilde{T}_{2}} \tilde{\vartheta}_{13}+\frac{1}{T_{1}} \tilde{\vartheta}_{13} ; h_{2}=-\frac{2}{T_{2}} \vartheta_{23}-\frac{3}{\tilde{T}_{2}} \tilde{\vartheta}_{13}-\frac{2}{T_{1}} \tilde{\vartheta}_{13} ; h_{3}=-\frac{3}{T_{2}} \vartheta_{23}-\frac{1}{\tilde{T}_{2}} \tilde{\vartheta}_{13} ; \\
a_{1}=T_{1}\left(T_{2}\right)^{2} ; a_{2}=T_{1}\left(\tilde{T}_{2}\right)^{2} ; \tilde{\vartheta}_{13}=\left(\tilde{L}_{1} L_{\Delta} \tilde{L}_{3}\right)^{-1}, \vartheta_{23}=\left(L_{2} L_{\Delta} L_{3}\right)^{-1} \text { y } \Delta_{3}=2 \Delta_{1}-\Delta_{2} .
\end{gathered}
$$

La ec. (16) se expresa de la forma:

$$
\eta^{(3)}\left(\Delta_{1}, \Delta_{2}\right)=\left\{\eta_{0}^{2}+W_{2} J\left(\Delta_{1}, \Delta_{2}\right)\right\}^{1 / 2}
$$

Con $J\left(\Delta_{1}, \Delta_{2}\right)=g_{1} \Delta_{1}+g_{2} \Delta_{2}+g_{3} \Delta_{1}^{2} \Delta_{2}+g_{4} \Delta_{1} \Delta_{2}^{2}+g_{5} \Delta_{1}^{3}+g_{6} \Delta_{2}^{3}$, donde los coeficientes $g_{j}\left(\Delta_{1}, \Delta_{2}\right)(\mathrm{j}=1, \ldots, 6)$ están dados por:

$$
\begin{gathered}
g_{1}=\left[\left(-3 \tilde{T}_{2}-T_{1}\right) \frac{\tilde{\vartheta}_{13}}{a_{2}}+\left(-2 T_{2}-T_{1}\right) \frac{\vartheta_{23}}{a_{1}}\right] ; g_{2}=\left[\left(\tilde{T}_{2}+T_{1}\right) \frac{\tilde{\vartheta}_{13}}{a_{2}}+\left(2 T_{2}+T_{1}\right) \frac{\vartheta_{23}}{a_{1}}\right] g_{3}=\left(-3 \tilde{\vartheta}_{13}-2 \vartheta_{23}\right) ; \\
g_{4}=\left(\tilde{\vartheta}_{13}+3 \vartheta_{23}\right) ; g_{5}=2 \vartheta_{13} ; \quad g_{6}=-\vartheta_{23} .
\end{gathered}
$$

Los puntos A, B, C, D están descritos por las siguientes coordenadas, de acuerdo a la Fig. 5:

$$
\begin{gathered}
A\left(\Delta_{1}, \Delta_{2}\right): \Delta_{1}=\Delta_{1}^{0}, \Delta_{2}=\Delta_{2}^{0} ; \\
\mathrm{B}\left(\Delta_{1}, \Delta_{2}\right): \Delta_{1}=\Delta_{1}^{0}, \Delta_{2}=2 \Delta_{1}^{0}-\Delta_{2}^{0} ; \\
\mathrm{C}\left(\Delta_{1}, \Delta_{2}\right): \Delta_{1}=-\Delta_{1}^{0}, \Delta_{2}=-2 \Delta_{1}^{0}+\Delta_{2}^{0} \\
\mathrm{D}\left(\Delta_{1}, \Delta_{2}\right): \Delta_{1}=-\Delta_{1}^{0}, \Delta_{2}=-\Delta_{2}^{0} .
\end{gathered}
$$

Estos puntos también pueden ser identificados en otro espacio $\tilde{\varepsilon}\left(\Delta_{1}, \Delta\right)$ de acuerdo a las siguientes coordenadas:

$$
A\left(\Delta_{1}, \Delta\right): \Delta_{1}=\Delta_{1}^{0}, \Delta=\Delta^{0} ; \mathrm{B}\left(\Delta_{1}, \Delta\right): \Delta_{1}=\Delta_{1}^{0}, \Delta=-\Delta^{0} ; \mathrm{C}\left(\Delta_{1}, \Delta\right): \Delta_{1}=-\Delta_{1}^{0}, \Delta=\Delta^{0} \mathrm{D}\left(\Delta_{1}, \Delta\right): \Delta_{1}=-\Delta_{1}^{0}, \Delta=-\Delta^{0} .
$$

Los puntos A, B, C, D también están caracterizados por los distintos procesos multi-fotónicos que tienen lugar, de acuerdo a la Fig. 6. Allí, hemos calculado el tamaño de la barra basándonos en las coordenadas asignadas a los puntos A, B, C, D, en una escala conforme al valor de la frecuencia $\omega_{0}$.

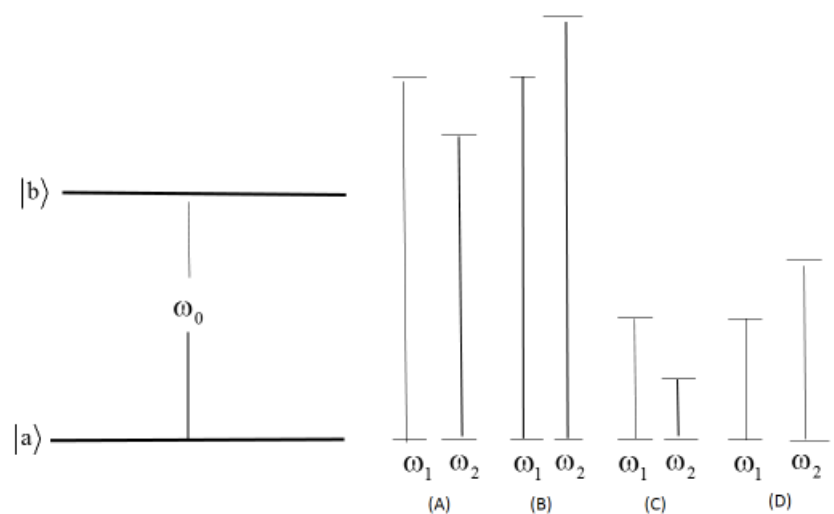

Fig. 6. Procesos fotónicos que distinguen cada uno de los puntos A,B,C,D. 
Esta representación de las frecuencias $\omega_{1}$ y $\omega_{2}$ en cada punto, permite explorar los diversos procesos que tienen lugar en la señal de mezcla de cuatro ondas y poder medir inclusive su eficiencia.

\section{Propiedades de simetrías en el espacio de frecuencias $\left(\Delta_{1}, \Delta_{2}\right)$}

Consideremos algunas propiedades de simetría tanto en la absorción como en el índice de refracción cuando nos desplazamos de un punto a otro, Fig. 5. Analicemos las distintas transformaciones considerando el punto A como punto de partida con las coordenadas ya mencionadas $\left(\Delta_{1}^{(0)}, \Delta_{2}^{(0)}\right)$. El coeficiente de absorción $\alpha^{(3)}\left(\Delta_{1}, \Delta_{2}\right)$, o el índice de refracción $\eta^{(3)}\left(\Delta_{1}, \Delta_{2}\right), \Delta_{1}$ y $\Delta_{2}$ son los tres ejes coordenados. En el punto A se puede definir:

$$
\begin{gathered}
\alpha_{(A)}^{(3)}\left(\Delta_{1}^{(0)}, \Delta_{2}^{(0)}\right)=\frac{W_{1}}{\eta_{(A)}^{(3)}\left(\Delta_{1}^{(0)}, \Delta_{2}^{(0)}\right)}\left[H_{(A)}\left(\Delta_{1}^{(0)}, \Delta_{2}^{(0)}\right)\right] \\
H_{(A)}\left(\Delta_{1}^{(0)}, \Delta_{2}^{(0)}\right)=h_{0}^{(0)}+h_{1}^{(0)} \Delta_{1}^{(0)} \Delta_{2}^{(0)}+h_{2}^{(0)}\left(\Delta_{1}^{(0)}\right)^{2}+h_{3}^{(0)}\left(\Delta_{2}^{(0)}\right)^{2} \\
h_{0}^{(0)}=\frac{1}{a_{1}} \vartheta_{23}^{(0)}+\frac{1}{a_{2}} \tilde{\vartheta}_{13}^{(0)} ; h_{1}^{(0)}=\frac{6}{T_{2}} \vartheta_{23}^{(0)}+\frac{4 T_{2}}{\tilde{T}_{2}} \tilde{T}_{2}^{(0)}+\frac{1}{T_{1}} \tilde{\vartheta}_{13}^{(0)} ; h_{2}^{(0)}=-\frac{2}{T_{2}} \vartheta_{23}^{(0)}-\frac{3}{\tilde{T}_{2}} \tilde{\vartheta}_{13}^{(0)}-\frac{2}{T_{1}} \tilde{\vartheta}_{13}^{(0)} \\
h_{3}^{(0)}=-\frac{3}{T_{2}} \vartheta_{23}^{(0)}-\frac{1}{\tilde{T}_{2}} \tilde{\vartheta}_{13}^{(0)} ; \text { donde } \tilde{\vartheta}_{13}^{(0)}=\left(\tilde{L}_{1}^{(0)} L_{\Delta}^{(0)} \tilde{L}_{3}^{(0)}\right)^{-1}, \vartheta_{23}^{(0)}=\left(L_{2}^{(0)} L_{\Delta}^{(0)} L_{3}^{(0)}\right)^{-1} .
\end{gathered}
$$

Para el índice de refracción en el punto (A) tenemos:

$$
\begin{gathered}
\eta_{(A)}^{(3)}\left(\Delta_{1}^{(0)}, \Delta_{2}^{(0)}\right)=\left\{\eta_{0}^{2}+W_{2} J_{(A)}\left(\Delta_{1}^{(0)}, \Delta_{2}^{(0)}\right)\right\}^{1 / 2} \\
J_{(A)}\left(\Delta_{1}^{(0)}, \Delta_{2}^{(0)}\right)=g_{1}^{(0)} \Delta_{1}^{(0)}+g_{2}^{(0)} \Delta_{2}^{(0)}+g_{3}^{(0)}\left(\Delta_{1}^{(0)}\right)^{2} \Delta_{2}^{(0)}+g_{4}^{(0)} \Delta_{1}^{(0)}\left(\Delta_{2}^{(0)}\right)^{2}+g_{5}^{(0)}\left(\Delta_{1}^{(0)}\right)^{3}+g_{6}^{(0)}\left(\Delta_{2}^{(0)}\right)^{3},
\end{gathered}
$$

donde los coeficientes $g_{j}\left(\Delta_{1}^{(0)}, \Delta_{2}^{(0)}\right)(\mathrm{j}=1, \ldots, 6)$ están dados por:

$$
\begin{gathered}
g_{1}^{(0)}=\left[\left(-3 \tilde{T}_{2}-T_{1}\right) \frac{\tilde{\vartheta}_{13}^{(0)}}{a_{2}}+\left(-2 T_{2}-T_{1}\right) \frac{\vartheta_{23}^{(0)}}{a_{1}}\right] ; g_{2}^{(0)}=\left[\left(\tilde{T}_{2}+T_{1}\right) \frac{\tilde{\vartheta}_{13}^{(0)}}{a_{2}}+\left(2 T_{2}+T_{1}\right) \frac{\vartheta_{23}^{(0)}}{a_{1}}\right] g_{3}^{(0)}=\left(-3 \tilde{\vartheta}_{13}^{(0)}-2 \vartheta_{23}^{(0)}\right) ; \\
g_{4}^{(0)}=\left(\tilde{\vartheta}_{13}^{(0)}+3 \vartheta_{23}^{(0)}\right) ; g_{5}^{(0)}=2 \tilde{\vartheta}_{13}^{(0)} ; g_{6}^{(0)}=-\vartheta_{23}^{(0)} .
\end{gathered}
$$

Considerando las coordenadas $\left(\Delta_{1}, \Delta_{2}\right)$ del punto (B), Fig. 5 , tenemos que la transformación desde A hasta B, definida como $T_{B A}$ es tal que $\vartheta_{23}^{(0)}$ permanece constante, mientras que $\tilde{\vartheta}_{13}^{(0)}$ se transforma en $\tilde{\vartheta}_{12}^{(0)}$, y por tanto los coeficientes $h_{k}^{(0)}$ se transforman en los coeficientes $u_{k}^{(0)}$, de manera que el coeficiente de absorción en el punto (B) queda definido como:

$$
\alpha_{(\mathrm{B})}^{(3)}\left(\Delta_{1}^{(0)}, \Delta_{2}^{(0)}\right)=\frac{W_{1}}{\eta_{(\mathrm{B})}^{(3)}\left(\Delta_{1}^{(0)}, \Delta_{2}^{(0)}\right)}\left[H_{(\mathrm{B})}\left(\Delta_{1}^{(0)}, \Delta_{2}^{(0)}\right)\right]
$$

donde ahora se tiene que:

$$
H_{(\mathrm{B})}\left(\Delta_{1}^{(0)}, \Delta_{2}^{(0)}\right)=\tilde{u}_{0}^{(0)}+\tilde{u}_{1}^{(0)} \Delta_{1}^{(0)} \Delta_{2}^{(0)}+\tilde{u}_{2}^{(0)}\left(\Delta_{1}^{(0)}\right)^{2}+\tilde{u}_{3}^{(0)}\left(\Delta_{2}^{(0)}\right)^{2},
$$

y allí los coeficientes quedan definidos como:

$$
\tilde{u}_{0}^{(0)}=u_{o}^{(0)} ; \tilde{u}_{1}^{(0)}=-\left(u_{1}^{(0)}+4 u_{3}^{(0)}\right) ; \tilde{u}_{2}^{(0)}=2 u_{1}^{(0)}+u_{2}^{(0)}+4 u_{3}^{(0)} ; \tilde{u}_{3}^{(0)}=u_{3}^{(0)},
$$

mientras que: 


$$
\begin{gathered}
\tilde{u}_{0}^{(0)}=\frac{1}{a_{1}} \vartheta_{23}^{(0)}+\frac{1}{a_{2}} \tilde{\vartheta}_{12}^{(0)} ; \tilde{u}_{1}^{(0)}=\frac{6}{T_{2}} \vartheta_{23}^{(0)}-\frac{4 T_{2}}{\tilde{T}_{2}} \tilde{T}_{12}^{(0)}-\frac{1}{T_{1}} \tilde{\vartheta}_{12}^{(0)}+\frac{1}{\tilde{T}_{2}} \tilde{\vartheta}_{12}^{(0)} ; \\
\tilde{u}_{2}^{(0)}=-\frac{2}{T_{2}} \vartheta_{23}^{(0)}+\frac{8 T_{2}}{\tilde{T}_{2} \tilde{T}_{2}} \tilde{\vartheta}_{12}^{(0)}-\frac{7}{\tilde{T}_{2}} \tilde{\vartheta}_{12}^{(0)} ; \tilde{u}_{3}^{(0)}=-\frac{3}{T_{2}} \vartheta_{23}^{(0)}-\frac{1}{\tilde{T}_{2}} \tilde{\vartheta}_{12}^{(0)}
\end{gathered}
$$

Para el índice de refracción tenemos en el punto (B):

$$
\eta_{(\mathrm{B})}^{(3)}\left(\Delta_{1}^{(0)}, \Delta_{2}^{(0)}\right)=\left\{\eta_{0}^{2}+W_{2} J_{(\mathrm{B})}\left(\Delta_{1}^{(0)}, \Delta_{2}^{(0)}\right)\right\}^{1 / 2}
$$

En este punto los $g_{j}^{(0)}$ se convierten en $\tilde{g}_{j}^{(0)}$, puesto que $\tilde{\vartheta}_{13}^{(0)}$ se transforma en $\tilde{\vartheta}_{12}^{(0)}$ y $\vartheta_{23}^{(0)}$ permanece constante.

$$
\begin{gathered}
J_{(\mathrm{B})}\left(\Delta_{1}^{(0)}, \Delta_{2}^{(0)}\right)=\tilde{\tilde{g}}_{1}^{(0)} \Delta_{1}^{(0)}+\tilde{\tilde{g}}_{2}^{(0)} \Delta_{2}^{(0)}+\tilde{\tilde{g}}_{3}^{(0)}\left(\Delta_{1}^{(0)}\right)^{2} \Delta_{2}^{(0)}+\tilde{\tilde{g}}_{4}^{(0)} \Delta_{1}^{(0)}\left(\Delta_{2}^{(0)}\right)^{2}+\tilde{\tilde{g}}_{5}^{(0)}\left(\Delta_{1}^{(0)}\right)^{3}+\tilde{\tilde{g}}_{6}^{(0)}\left(\Delta_{2}^{(0)}\right)^{3}, \\
\tilde{\tilde{g}}_{1}^{(0)}=\tilde{g}_{1}^{(0)}+2 \tilde{g}_{2}^{(0)} ; \tilde{\tilde{g}}_{2}^{(0)}=g_{2}^{(0)} ; \tilde{\tilde{g}}_{3}^{(0)}=\tilde{g}_{3}^{(0)}+4 \tilde{g}_{4}^{(0)}+12 \tilde{g}_{6}^{(0)} ; \tilde{\tilde{g}}_{4}^{(0)}=\tilde{g}_{4}^{(0)}+6 g_{6}^{(0)} ; \tilde{\tilde{g}}_{5}^{(0)}=2 \tilde{g}_{3}^{(0)}+4 \tilde{g}_{4}^{(0)}+\tilde{g}_{5}^{(0)}+8 \tilde{g}_{6}^{(0)} ; \\
\tilde{\tilde{g}}_{6}^{(0)}=g_{6}^{(0)} .
\end{gathered}
$$

Para el punto C de la Fig. 5 podemos ver que:

$$
\alpha_{(\mathrm{C})}^{(3)}\left(\Delta_{1}^{(0)}, \Delta_{2}^{(0)}\right)=\frac{W_{1}}{\eta_{(\mathrm{C})}^{(3)}\left(\Delta_{1}^{(0)}, \Delta_{2}^{(0)}\right)}\left[H_{(\mathrm{C})}\left(\Delta_{1}^{(0)}, \Delta_{2}^{(0)}\right)\right]
$$

donde se cumple que $H_{(\mathrm{C})}\left(\Delta_{1}^{(0)}, \Delta_{2}^{(0)}\right)=\tilde{u}_{0}^{(0)}+\tilde{u}_{1}^{(0)} \Delta_{1}^{(0)} \Delta_{2}^{(0)}+\tilde{u}_{2}^{(0)}\left(\Delta_{1}^{(0)}\right)^{2}+\tilde{u}_{3}^{(0)}\left(\Delta_{2}^{(0)}\right)^{2}$, en vista de que $\tilde{\vartheta}_{13}^{(0)}$ se transforma en $\tilde{\vartheta}_{12}^{(0)}$ y $\vartheta_{23}^{(0)}$ permanece constante. Para el índice de refracción no lineal tenemos:

$$
\eta_{(\mathrm{C})}^{(3)}\left(\Delta_{1}^{(0)}, \Delta_{2}^{(0)}\right)=\left\{\eta_{0}^{2}+W_{2} J_{(\mathrm{C})}\left(\Delta_{1}^{(0)}, \Delta_{2}^{(0)}\right)\right\}^{1 / 2},
$$

donde es posible demostrar que $J_{(\mathrm{C})}\left(\Delta_{1}^{(0)}, \Delta_{2}^{(0)}\right)=-J_{(\mathrm{B})}\left(\Delta_{1}^{(0)}, \Delta_{2}^{(0)}\right)$. Finalmente, en el punto D de la Fig. 5 observamos que:

$$
\alpha_{(\mathrm{D})}^{(3)}\left(\Delta_{1}^{(0)}, \Delta_{2}^{(0)}\right)=\frac{W_{1}}{\eta_{(\mathrm{D})}^{(3)}\left(\Delta_{1}^{(0)}, \Delta_{2}^{(0)}\right)}\left[H_{(\mathrm{D})}\left(\Delta_{1}^{(0)}, \Delta_{2}^{(0)}\right)\right]
$$

$H_{(\mathrm{D})}\left(\Delta_{1}^{(0)}, \Delta_{2}^{(0)}\right)=h_{0}^{(0)}+h_{1}^{(0)} \Delta_{1}^{(0)} \Delta_{2}^{(0)}+h_{2}^{(0)}\left(\Delta_{1}^{(0)}\right)^{2}+h_{3}^{(0)}\left(\Delta_{2}^{(0)}\right)^{2}$. En este punto $\tilde{\vartheta}_{13}^{(0)}$ y $\vartheta_{23}^{(0)}$ permanecen constantes, por lo que los coeficientes $g_{j}\left(\Delta_{1}^{(0)}, \Delta_{2}^{(0)}\right)(\mathrm{j}=1, \ldots, 6)$ permanecen constantes, y el índice de refracción en este caso es,

$$
\eta_{(\mathrm{D})}^{(3)}\left(\Delta_{1}^{(0)}, \Delta_{2}^{(0)}\right)=\left\{\eta_{0}^{2}+W_{2} J_{(\mathrm{D})}\left(\Delta_{1}^{(0)}, \Delta_{2}^{(0)}\right)\right\}^{1 / 2} ;
$$

es posible demostrar que $J_{(\mathrm{D})}\left(\Delta_{1}^{(0)}, \Delta_{2}^{(0)}\right)=-J_{(\mathrm{A})}\left(\Delta_{1}^{(0)}, \Delta_{2}^{(0)}\right)$. Con estas consideraciones se tienen las siguientes relaciones:

$$
\begin{aligned}
& \alpha_{(\mathrm{B})}^{(3)} \eta_{(\mathrm{B})}^{(3)}=\alpha_{(\mathrm{C})}^{(3)} \eta_{(\mathrm{C})}^{(3)} \quad \alpha_{(\mathrm{B})}^{(3)} \eta_{(\mathrm{B})}^{(3)} \neq \alpha_{(\mathrm{A})}^{(3)} \eta_{(\mathrm{A})}^{(3)} \quad \alpha_{(\mathrm{C})}^{(3)} \eta_{(\mathrm{C})}^{(3)} \neq \alpha_{(\mathrm{A})}^{(3)} \eta_{(\mathrm{A})}^{(3)} \\
& \alpha_{(\mathrm{D})}^{(3)} \eta_{(\mathrm{D})}^{(3)}=\alpha_{(\mathrm{A})}^{(3)} \eta_{(\mathrm{A})}^{(3)} \quad \alpha_{(\mathrm{D})}^{(3)} \eta_{(\mathrm{D})}^{(3)} \neq \alpha_{(\mathrm{C})}^{(3)} \eta_{(\mathrm{C})}^{(3)} \quad \alpha_{(\mathrm{B})}^{(3)} \eta_{(\mathrm{B})}^{(3)} \neq \alpha_{(\mathrm{D})}^{(3)} \eta_{(\mathrm{D})}^{(3)} \quad \alpha_{(\mathrm{A})}^{(3)} \eta_{(\mathrm{A})}^{(3)}+\alpha_{(\mathrm{C})}^{(3)} \eta_{(\mathrm{C})}^{(3)}=\alpha_{(\mathrm{B})}^{(3)} \eta_{(\mathrm{B})}^{(3)}+\alpha_{(\mathrm{D})}^{(3)} \eta_{(\mathrm{D})}^{(3)} \\
& \alpha_{(\mathrm{A})}^{(3)} \eta_{(\mathrm{A})}^{(3)}+\alpha_{(\mathrm{B})}^{(3)} \eta_{(\mathrm{B})}^{(3)}=\alpha_{(\mathrm{C})}^{(3)} \eta_{(\mathrm{C})}^{(3)}+\alpha_{(\mathrm{D})}^{(3)} \eta_{(\mathrm{D})}^{(3)}
\end{aligned}
$$

Por tanto, si se analizan las propiedades antes indicadas, y se recuerda que $\Delta=\Delta_{1}-\Delta_{2}$, es posible concluir lo que sigue. 
En los desplazamientos del punto A al B y del punto C al D, los valores de los productos $\alpha^{(3)} \eta^{(3)}$ no mantienen la misma magnitud. En ambos casos se establece una reflexión en el plano perpendicular al eje $\Delta_{2}$ que intersecta al eje $\Delta_{1}=\Delta_{2}$, (manteniendo $\Delta_{1}$ constante), con el cambio de $\Delta$ a $-\Delta(A \rightarrow B)$. Para la transformación $(C \rightarrow D),-\Delta_{1}$ se mantiene constante con cambio de $\Delta$ por $-\Delta$. Ambos casos corresponden a una reflexión en el diagrama para una pendiente +1 en el caso cuando $\Delta_{1}=\Delta_{2}$.

De igual forma encontramos cambios en la magnitud del producto $\alpha^{(3)} \eta^{(3)}$ al pasar del punto A al C y del B al $\mathrm{D}$, donde tenemos cambio de $\Delta_{1}$ a $-\Delta_{1}$, manteniendo el valor de $\Delta$ constante $\left(A \rightarrow C\right.$ ) y cambio de $\Delta_{1}$ a $-\Delta_{1}$, manteniendo $-\Delta$ constante $\left(B \rightarrow D\right.$ ). Esto último se traduce en una reflexión en el plano $\Delta_{2}$ seguida de una rotación $C_{2}\left(180^{\circ}\right)$ en torno al eje $\Delta_{1}$. A diferencia de esto, el producto $\alpha^{(3)} \eta^{(3)}$ se mantiene constante cuando hace una inversión del punto A al D y del punto $\mathrm{B}$ al $\mathrm{C}$, lo cual se traduce en cambio de $\Delta_{1}$ a $-\Delta_{1}$ y de $\Delta$ a $-\Delta(A \rightarrow D)$ y de $\Delta_{1}$ a $-\Delta_{1}$ y $-\Delta$ a $\Delta(B \rightarrow C)$, respectivamente. Es posible que las relaciones (23) puedan expresarse sólo en términos del coeficiente de absorción, cuando sean válidas las condiciones del tipo $\left(W_{2} J_{(k)} / \eta_{0}^{2}\right) \ll 1(\mathrm{k}=\mathrm{A}, \mathrm{B}, \mathrm{C}, \mathrm{D})$, haciendo el $\eta_{(k)}^{(3)} \approx \eta_{0}=1.333$ al usar como solvente el agua.

\section{Comentarios finales}

Hemos estudiado el comportamiento de la señal de mezcla de cuatro ondas en el espacio de frecuencias. El modelo corresponde a un sistema de dos estados bajo interacción aleatoria con el reservorio térmico, y con interacción radiativa modelada en forma perturbativa hasta el tercer orden en las amplitudes de los campos electromagnéticos. El estudio presentado aquí corresponde a definir propiedades de simetría inherentes a los perfiles en el espacio de frecuencias, tanto para el coeficiente de absorción no lineal como para el índice de refracción no lineal de sistemas moleculares complejos. Para particularizar el estudio, hemos seleccionado como sistema molecular el colorante orgánico Malaquita Verde dado el conocimiento pleno existente de los parámetros que lo caracterizan, además de que posee una respuesta altamente resonante. Seleccionamos la señal de mezcla de cuatro ondas y propagamos la misma a lo largo del camino óptico, para dar cuenta de las propiedades ópticas. Definimos espacios tridimensionales conformados por la propiedad óptica $\alpha$ o $\eta$ y las variables $\Delta_{1}, \Delta_{2}$, para el estudio de las propiedades de simetría. Una de ellas correspondiente a la inversión respecto al origen de coordenadas y las dos restantes de reflexión en los planos $\Delta_{1}, \Delta_{2}$ correspondientes. Hemos observado de los cálculos realizados, que en la inversión se muestra claramente la asimetría en la magnitud del coeficiente de absorción. De igual forma, pero con diferencias más pequeñas entre los valores de los coeficientes de absorción, se observan en los casos de transformaciones que puedan ser vistas como reflexiones en los planos de estudio. Este tipo de simetrías inherentes pueden ser de considerable interés cuando se intente medir normalmente, a partir de los perfiles de absorción, los tiempos de relajación en el semi-ancho a la semi-altura (HWHM) de la distribución. Finalmente, como se observa en la Fig. 3, es importante resaltar el hecho que aunque los puntos de simetrías A y B son equivalentes a los puntos C y D en el mapa de contorno, los procesos fotónicos que rigen cada pareja son muy distintos. Como pudo observarse en el análisis, es algo complicado disociar las dos propiedades ópticas, dado que el comportamiento en la propiedad de absorción está inversamente relacionada con el de refracción, a diferencia de este último cuyo comportamiento depende exclusivamente de sí mismo.

\section{Agradecimientos}

JLP y JFC agradecen a la Secretaría Nacional de Ciencia, Tecnología e Innovación (SENESCYT) por el financiamiento brindado a través del Proyecto Becas Prometeo 